Received September 28, 2021; Accepted November 22, 2021;

Published online on November 26, 2021

DOI: 10.2116/analsci.21P287

\title{
Microwave-Assisted Facile Synthesis of N, P Co-doped Fluorescent Carbon Dot Probe
}

\section{for Determination of Nifedipine}

\author{
Saeedeh Narimani**, Naser Samadi ${ }^{a}$, Tooba Hallaj ${ }^{\mathrm{b}}$ \\ ${ }^{a}$ Department of Analytical Chemistry, Faculty of Chemistry, Urmia University, Urmia, Iran \\ ${ }^{b}$ Cellular and Molecular Research Center, Cellular and Molecular Medicine Institute, Urmia \\ University of Medical Sciences, Urmia 5714783734, Iran
}

Emails: Saeedeh.narimani@gmail.com,

samadi76@yahoo.com,t.hallaj@tabrizu.ac.ir

*Corresponding author: Saeedeh.narimani@gmail.com

Telephone: (+98)9147242335 


\begin{abstract}
:
A simple and fast microwave synthesis method was applied for the preparation of several carbon dots (CDs) from various combinations of urea, phosphoric acid, and B-alanine as nitrogen, phosphorus, and carbon precursors. The maximum quantum yield (44\%) was obtained for nitrogen and phosphorus codoped carbon dots (N, P-CDs) prepared from urea, B-alanine, and phosphoric acid. Furthermore, N, PCDs were exploited to synthesize a simple and sensitive fluorometric probe to determine of nifedipine (NFD). We indicated that the analytical response of the designed sensor could be affected by the kind of dopant and synthesis precursors. It is worth mentioning that just the fluorescence intensity of N, PCDs was declined by NFD, and no fluorescence quenching was observed for other prepared CDs. The NFD-developed sensor indicated the linear response range of $3.3 \times 10^{-8}$ to $3.2 \times 10^{-5} \mathrm{~mol} / \mathrm{L}$, with the detection limit of $1.0 \times 10^{-8} \mathrm{~mol} / \mathrm{L}$. The sensor was successfully applied to measure NFD in human biological fluids.
\end{abstract}

Keywords: Fluorescence; N, P-CDs; Microwave synthesis; Nifedipine.

\title{
1. Introduction
}

Nifedipine (NFD) is a dihydropyridine channel blocker for calcium that impedes the entry of calcium particles through layers into the cardiovascular smooth muscle cells. NFD causes dilation of coronary arteries and prevents calcium contraction and vasoconstriction utilized in treating of high blood pressure, angina pectoris, and some other cardiovascular disorders ${ }^{1-3}$. However, the overdose of NFD leads to diseases such as palpitations, dizziness and may cause vomiting due to its toxicity ${ }^{4,5}$. Therefore, monitoring of NFD in human biological fluids is essential. Different methods, 
including chromatographic ${ }^{6-8}$, electrochemical ${ }^{9,10}$, and spectroscopy ${ }^{11,12}$, have been reported for the quantification of NFD. Some of these methods are expensive, complicated, and time-consuming. The fluorometric techniques have been applied for designing simple, sensitive, and selective sensors for the measurement of different compounds in biological fluid. In these sensors, diverse phenomena such as dynamic and static quenching, inner filter effect (IFE), photoinduced electron transfer (PET), and energy transfer have been introduced as the quenching mechanism ${ }^{13}$.

Recently, carbon dots (CDs) have been applied for designing various tune-off fluorometric sensors for the determination of different compounds including nifedipine ${ }^{14-18}$. CDs have received particular attention due to their high biocompatibility, photostability, water solubility, and tunable excitation and emission spectra ${ }^{19-21}$. These nanoparticles were synthesized by several methods such as hydrothermal $^{22}$, solvothermal ${ }^{23,24}$, ultrasonication ${ }^{25,26}$, and microwave, which microwave method has attracted additional notice due to its simplicity and rapidness compared to others ${ }^{27-29}$.

The fluorescence features of CDs can be affected by various factors such as their size distribution, passivation agents, dopant, solvent, $\mathrm{pH}$, and so on ${ }^{30,31}$. One of the strategies to improve the fluorescence properties of CDs is heteroatom doping processes. This process can alter the chemical and optical properties of CDs and extend their potential applications ${ }^{32-34}$. The most common heteroatom that has been widely used for doping CDs is nitrogen. Concerning the synergistic effect of different elements, co-doping of nitrogen and various heteroatoms such as boron, sulfur, and phosphorus, have been used to improve the quantum yield and selectivity of CDs towards appropriate applications ${ }^{34-38}$.

It is worth mentioning that the sensitivity and selectivity of doped CDs toward the determination of analytes is different from the un-doped CDs. For instance, a boron-doped-CDs was used as fluorescent probe to determine triticonazole in which triticonazole quenched the fluorescence intensity of boron-doped CDs, while it has no effect on the un-doped CDs ${ }^{39}$. In the other study, a selective $\mathrm{S}, \mathrm{N}$-doped CDs based fuorescence probe was designed for the determination of nitric oxide, which the selectivity of this sensor was attributed to doping of $\mathrm{S}$ atoms ${ }^{40}$. 
Herein, a selective and simple CDs-based fluorometric probe was designed for analysis of NFD. Various single and co-doped CDs were prepared by a fast and one-step microwave-assisted synthesis method. The effect of dopant precursors was studied on the selectivity of the designed sensor toward NFD. N, P co-doped CDs were chosen as the best candidate to develop a turn-off fluorometric sensor for the determination of NFD in the real human samples.

\section{Materials and methods}

\section{Chemicals and materials}

All reagents were of analytical grade. Potassium dihydrogen phosphate, dipotassium hydrogen phosphate, Urea, B-alanine, phosphoric acid, nifedipine, amino acids, and quinine sulfate dihydrate were purchased from Merck. Deionized water was utilized for all experiments.

\section{Apparatus}

FT-IR spectra were recorded by a (Bruker Co., Germany, www. bruker. com) Tensor-27 FT-IR spectrometer. UV-Vis absorption spectra were performed on a (Varian, Australia) Cary-100 spectrophotometer. JASCO FP-8300 fluorescence spectrophotometer was used for recording the fluorescence intensity. All measurements were done in a standard $1.0 \mathrm{~cm}$ quartz cell. $\mathrm{pH}$ was measured on a Metrohm 827 PH-Lab. The shape and particle size of the synthesized CDs were determined by Transmission electron microscopy (TEM) in a (TEM, Zeiss, Leo 906, Germany) model that the voltage was $100 \mathrm{kV}$. Furthermore, the X-ray diffraction (XRD) pattern was performed with a Philips PW 1730 diffractometer at a voltage of $40 \mathrm{kV}$. The XPS analyses were conducted on a Specs-Flex $\mathrm{X}$-ray photoelectron spectroscope with the energy step size between $200 \mathrm{eV}$ to $4 \mathrm{KeV}$.

\section{Preparation procedure for $\mathrm{N}, \mathrm{P}-\mathrm{CD}$}

$\mathrm{N}, \mathrm{P}$-CDs were synthesized via a fast and one-step microwave method. First, $1.5 \mathrm{~g}$ urea, $1 \mathrm{~g}$ B-alanine and $4 \mathrm{~mL} \mathrm{H} \mathrm{H}_{3} \mathrm{PO}_{4}$ were mixed, and dissolved in $20 \mathrm{~mL}$ deionized water and stirred vigorously to form a clear and homogeneous solution. The mixture was put into a $900 \mathrm{~W}$ microwave oven for $3.5 \mathrm{~min}$. The 
obtained brown solid product was dissolved in $20 \mathrm{~mL}$ deionized water with stirring. To expel large particles, the solution was centrifuged at $6000 \mathrm{rpm}$ for $20 \mathrm{~min}$, and then filtered with $0.22 \mu \mathrm{m}$ cellulose acetate microporous filter membranes. Finally, the obtained $\mathrm{CDs}$ were kept at $4^{\circ} \mathrm{C}$ for further use.

\section{General procedure for measurement of nifedipine}

Generally, $4 \mu \mathrm{L}$ of CDs solution, $500 \mu \mathrm{L}$ of $0.02 \mathrm{~mol} / \mathrm{L}$ phosphate buffer solution ( $\mathrm{pH}=6.0$ ), and specific concentration of NFD standard solution were added to a quartz cell. After the addition of deionized water, the volume of the solution was reached $1.5 \mathrm{~mL}$. Then, the FL spectra were immediately recorded at $402 \mathrm{~nm}$ when the excitation wavelength was $365 \mathrm{~nm}$ at room temperature. The selectivity of the method toward NFD was also determined with the addition of coexistent materials in a similar manner in the presence of NFD. All the experiments were done in triplicate.

\section{Preparation procedure for real sample analysis}

Plasma samples were acquired from Tabriz Blood Transfusion Center (Iran). A $500 \mu \mathrm{L}$ of real plasma was spiked with a specific concentration of NFD standard solution in a test tube. Afterwards $500 \mu \mathrm{L}$ of acetonitrile was added into the tube, and a centrifuge was performed at $6000 \mathrm{rpm}$ for $35 \mathrm{~min}$ to precipitate the plasma proteins. Then, the supernatant solution was separated and reached $10 \mathrm{~mL}$ with deionized water. An appropriate volume of this solution was detected. The urine samples were obtained from healthy volunteers and spiked with proper concentration of NFD, and then centrifuge was performed at $6000 \mathrm{rpm}$ for $25 \mathrm{~min}$. After the dilution of the sample up to 4 times with deionized water, the specific amounts of the solution were used for subsequent experiments.

\section{Results and discussion}

\section{Structural characterization of CDs}

A novel, simple and straightforward microwave synthesis method was used to prepare co-doped CDs from urea, phosphoric acid, and B-alanine as nitrogen, phosphorus, and carbon sources, respectively. The as-synthesized N, P-CDs were characterized by various techniques. TEM image displayed N, P- 
CDs have uniform dispersions without apparent aggregation and have spherical shapes. The N, P-CDs are mainly distributed in the range of 6-8 nm (Fig. 1). As shown in the inset of Fig. 1, these CDs (which have light yellow color under visible light) exhibited a strong bright blue color under $365 \mathrm{~nm}$ UV light illumination. XRD pattern of the CDs showed a broad diffraction peak located at $2 \theta=24.9^{\circ}$, which is attributed to the graphitic structure of the N, P-CDs ${ }^{41}$ (Fig. S1, Supporting Information). The functional groups on the surface of prepared CDs were distinguished by FT-IR (Fig. S2). A peak that appeared between $3000 \mathrm{~cm}^{-1}$, and $3400 \mathrm{~cm}^{-1}$ is ascribed to stretching vibration of hydroxyl, carboxylic, and $-\mathrm{NH}$ groups, and the peak at $1659 \mathrm{~cm}^{-1}$ is ascribed to a $\mathrm{C}=\mathrm{O}$ stretching ${ }^{42,43}$. The stretching vibration bands of $\mathrm{CH}_{2}$ has appeared at $2438 \mathrm{~cm}^{-1}$. Furthermore, the peaks at $1448 \mathrm{~cm}^{-1}, 1402 \mathrm{~cm}^{-1}, 1111$ $\mathrm{cm}^{-1}$, and $992 \mathrm{~cm}^{-1}$ are ascribed to P-C, $-\mathrm{COO}, \mathrm{C}-\mathrm{O}-\mathrm{P}$, and C-O stretching vibrations, respectively. The XPS were utilized further to confirm the functional groups and elements of N, P-CDs. The XPS survey spectra shown in Fig. 2a indicate three prominent peaks: C $1 \mathrm{~s}(282 \mathrm{eV}), \mathrm{O} 1 \mathrm{~s}(530 \mathrm{eV}), \mathrm{P} 2 \mathrm{p}(130 \mathrm{eV})$, and N1s (398). In the high-resolution spectrum of C $1 \mathrm{~s}$ (Fig. 2b), the three fitted peaks at 282.07, 282.28, and 285.27 are due to the presence of $\mathrm{C}=\mathrm{C}, \mathrm{C}-\mathrm{C}$, and $\mathrm{C}-\mathrm{O} / \mathrm{C}-\mathrm{N}$, respectively. The two peaks in the O1s high-resolution spectrum (Fig. 2c) are assigned to carbonyl (529.66 eV, C = O), and hydroxyl (C-O, or C-OH $530.37 \mathrm{eV}$ ). Moreover, the N1s survey (Fig. 2d) scan shows peaks at $397.84 \mathrm{eV}, 398.23$ $\mathrm{eV}$, and $399.79 \mathrm{eV}$, corresponding to the nitrogen in the form of $\mathrm{C}-\mathrm{N}-\mathrm{C}$, nitrogen in the form of $\mathrm{N}-(\mathrm{C})_{3}$ and $\mathrm{H}_{2} \mathrm{~N}-\mathrm{C}$, respectively. The high-resolution $\mathrm{P} 2 \mathrm{p}$ spectrum contains two peaks at 131.30 and 132.17 assigned to $\mathrm{P}=\mathrm{O}$ and $\mathrm{P}-\mathrm{C}$ groups (Fig. 2e) ${ }^{42,44,45}$. The results showed that the as-prepared CDs have hydrophilic surfaces due to the amino, hydroxyl, carboxylic, and carbonyl groups on their surface, which make the CDs highly water-soluble and enable them to be applied in fluorescent-based applications and sensors. Also, XPS and FT-IR obtained results confirmed that the as-prepared CDs were successfully doped with nitrogen and phosphorus heteroatoms. Furthermore, the UV-vis absorption and fluorescence emission spectra of the N, P-CDs were exhibited in Fig. S3. The two peaks at $252 \mathrm{~nm}$ and 324 in the UV-vis absorption spectrum of $\mathrm{N}, \mathrm{P}-\mathrm{CDs}$ are attributed to the transition $\pi-\pi^{*}$ of $\mathrm{C}=\mathrm{C}$ bonds of carbon core and transition $\mathrm{n}-\pi^{*}$ of carbonyl, respectively. Moreover, the fluorescence intensity at different excitations and the 3D fluorescence plot of the CDs with a variation of the emission 
and excitation wavelengths were recorded. Fig. S4 showed that the synthesized N, P- CDs were indicated excitation-dependent emission behavior, and the maximum fluorescence emission was obtained at $365 / 402 \mathrm{~nm}(\lambda \mathrm{ex} / \lambda \mathrm{em})$. Therefore, $365 \mathrm{~nm}$ was selected as the optimum excitation wavelength in all experiments. Furthermore, QY of N, P-CDs was estimated to be $44 \%$.

\section{N, P-CD probe for NFD sensing}

To design a sensitive probe for the detection of NFD, we investigated the effect of dopants and their synthesis sources on the fluorescence signal of CDs in the presence and absence of NFD. For this purpose, various precursors, including citric acid, glucose, urea, B-alanine, and $\mathrm{H}_{3} \mathrm{PO}_{4}$, were applied to the synthesis eight kinds of CDs (un-doped, single and co-doped CDs). The QY\% of all prepared CDs were reported in Table S1 (Supporting Information). N, P-CDs prepared from urea, B-alanine and $\mathrm{H}_{3} \mathrm{PO}_{4}$ indicated the highest QY\%. As shown in Fig. 3a, these N, P-CDs exhibited the high-intensity blue emission under $365 \mathrm{~nm}$ UV light. The results confirmed the formation of radiative relaxation pathways and improvement of the photophysical properties of CDs due to co-doping of $\mathrm{N}$ and $\mathrm{P}$. Then, the quenching effect of NFD was studied on the eight as-prepared CDs. Fig. 3b showed that the fluorescence intensity of N, P-CDs significantly declined by NFD while this drug indicated no declined effect on the other CDs. We suspected that because of existing n-type dopants, $\mathrm{N}$ and $\mathrm{P}$, in the structure of N, P-CDs, the electron concentration of N, P-CDs is high. On the other hand, as a result of the nitro group that is present in the structure of NFD structure, it can act as an electron acceptor toward N, PCDs. The NFD UV-vis absorption band is close to the bandgap of the as-prepared N, P-CDs (Fig. S5). Therefore, the electron can transfer from the conduction band of the N, P-CDs to the lowest unoccupied molecular orbital of NFD. Thus, the fluorescence quenching of CDs by NFD can be attributed to the electron transition processes between N, P-CDs, and NFD. Conversely, since the UV-vis spectrum of the NFD had no spectral overlap with the emission spectrum of the N, P-CDs, the quenching mechanism of N, P-CDs is not energy transfer Fig S6. Based on the unique behavior of N, P-CDs toward NFD, it was exploited to develop a fluorescence turn-off probe for the determination of NFD. 


\section{Analytical performance}

To enhance the sensitivity of the N, P-CDs for the determination of NFD, different variables that affected the interaction between NFD and CDs such as the amount of CDs, $\mathrm{pH}$, concentrations of buffer, and incubation time were explored on the fluorescence signal and the results were shown at Fig. S7.

After optimizing the experimental conditions, the experimental performance of the method for quantitative detection of NFD was assessed. The fluorescence intensity at $402 \mathrm{~nm}$ was continuously decliend with increasing the concentration of NFD due to the quenching of fluorescence (Fig. 4a). The fluorescence quenching can be depicted by Stern-Volmer's equation:

$$
\frac{F 0}{F}=1+k s v[c]
$$

$\mathrm{F}_{0}$ represents the fluorescence intensity of the fluorophore in the absence of a quencher, and $\mathrm{F}$ is the fuorescence intensity of fluorophore in the absence of a quencher, $\mathrm{K}_{\mathrm{SV}}$ represents the dynamic quenching constant, and $\mathrm{C}$ represents the concentration of the quencher.

As shown in Fig. 4b, the variation of fluorescence-quenching ratio $\left(\mathrm{F}_{0} / \mathrm{F}\right)$ versus the concentration of NFD indicated an excellent linearity in the concentration range of $3.3 \times 10^{-8}-3.2 \times 10^{-5} \mathrm{~mol} / \mathrm{L}$ with the detection limit of $1.0 \times 10^{-8} \mathrm{~mol} / \mathrm{L}$. Furthermore, (Table S2) indicates the comparison between the present method with some previously reported methods for NFD detection. The results show that the analytical performance of developed sensor is better than other methods, as well as this sensor is more costeffective than most methods.

\section{Study of interferences}

To evaluate the selectivity of the introduced method in the determination of NFD, the effect of some common interfering substances, including ions and compounds, which may be present in the real samples, was investigated on the analytical signal in the determination of NFD. The acceptable limits for interferences (the ratio of interfering substances concentration to the analyte that produces relative error $=5 \%$ or less) were reported in Table S3. As shown in Table S3, most compounds at high 
concentrations have no significant influence on the fluorescence signal. These results confirmed that the recommended sensor has sufficient selectivity for the measurement of NFD in real samples.

\section{Real sample measurements}

Human urine and plasma samples were applied to assess the practicability and reliability of the present method for the determination of NFD in real sample analyses. To evaluation of the developed method accuracy, appropriate amounts of NFD were spiked into the samples for analysis. The results were calculated and summarized in Table 1 . The recovery and RSD of the sensor were in the range of 95$105 \%$ and lower than $3.4 \%$, respectively. The results revealed that this method had good accuracy and is reliable for detecting NFD in real samples.

\section{Conclusion}

In summary, the present study aimed to develop a sensitive fluorometric sensor based on doped CDs with excellent water solubility through a facile and rapid one-step method to analyze of NFD. For this purpose, several CDs with different combinations precursors were prepared. N, P-CDs showed brilliant blue FL in exposing to UV light and possess a QY of $44 \%$. The fluorescence intensity of N, P-CDs effectively declined by the addition of NFD while this drug indicated a negligible effect on the other CDs. We noted that the analytical application of CDs could be affected by the kind of dopant and its precursors. The fluorescence quenching of N, P-CDs by NFD can be ascribed to the electron transition

processes between N, P-CDs, and NFD. Based on these facts, the simple and sensitive turn-off fluorescence nanoprobe was developed to measure NFD. The sensing system had remarkable advantages such as high speed, good selectivity, and wide response range, and it was used to detect NFD in human plasma and urine samples with satisfactory results.

Funding: This research did not receive any specific grant from funding agencies in the public, commercial, or profit sectors. 
Conflict of interest: The authors declare that they have no conflict to declare.

Table 1 Determination of NFD in human plasma and urine samples.

\begin{tabular}{|c|c|c|c|c|c|}
\hline Sample & $\begin{array}{l}\text { Added } \\
\left.{ }^{7} \mathrm{M}\right)\end{array}$ & $\begin{array}{l}\text { Found }\left(10^{-7} \mathrm{M}\right) \\
\text { a }\end{array}$ & Recoveries (\%) & $\operatorname{RSD}(\%, \mathrm{n}=3)$ & t-Statistics ${ }^{b}$ \\
\hline \multirow[t]{3}{*}{ Plasma1 } & 7.5 & 7.31 & 97.4 & 3 & 1.6 \\
\hline & 6.5 & 6.22 & 95.6 & 3.3 & \\
\hline & & & & & 2.3 \\
\hline \multirow[t]{2}{*}{ Plasma2 } & 7.5 & 7.9 & 105.3 & 2.6 & 3.3 \\
\hline & 6.5 & 6.25 & 96.1 & 2.4 & 2.8 \\
\hline \multirow[t]{3}{*}{ Urine 1} & 5 & 5.01 & 100.2 & 3.3 & 0.1 \\
\hline & 8 & 7.97 & 99.6 & 2.2 & \\
\hline & & & & & 0.3 \\
\hline \multirow[t]{2}{*}{ Urine 2} & 5 & 4.73 & 94.6 & 2.4 & 0.4 \\
\hline & 8 & 7.65 & 95.6 & 3.2 & 2.4 \\
\hline
\end{tabular}

a Mean of three determinations \pm standard deviation

b t-Critical $=4.3$ for $n=2, P=0.05$ 


\section{Caption to Figures}

Fig 1 TEM images of the synthesized N, P-CDs. Inset: Photograph of N, P-CDs under daylight (a) and the excitation of UV light with $365 \mathrm{~nm}$ (b).

Fig 2 (a) XPS spectra of CDs and the corresponding high-resolution XPS of the (b) C1s, (c) N1s, (d) O1s, and (e) P2p peak.

Fig 3 Fluorescence intensity of different CDs under excitation of UV light with $365 \mathrm{~nm}$ (a) and Fluorescence intensity of other CDs in the presence and absence of NFD (b), the concentration of NFD was $(60 \mu \mathrm{M}) . \mathrm{F}_{0}$ : fluorescence intensity of CDs in the absence of NFD, F: fluorescence intensity of CDs in the presence of NFD.

Fig 4 (a) Fluorescence spectra of the N, P-CDs upon addition of different concentrations of NFD $\left(3.3 \times 10^{-8}\right.$ to $\left.3.2 \times 10^{-5} \mathrm{M}\right)$. (b) The linear response of quenching effect $\left(\mathrm{F}_{0} / \mathrm{F}\right)$ of N, P-CDs $v$. the NFD concentration. (Excitation wavelength is $365 \mathrm{~nm}$ ). 


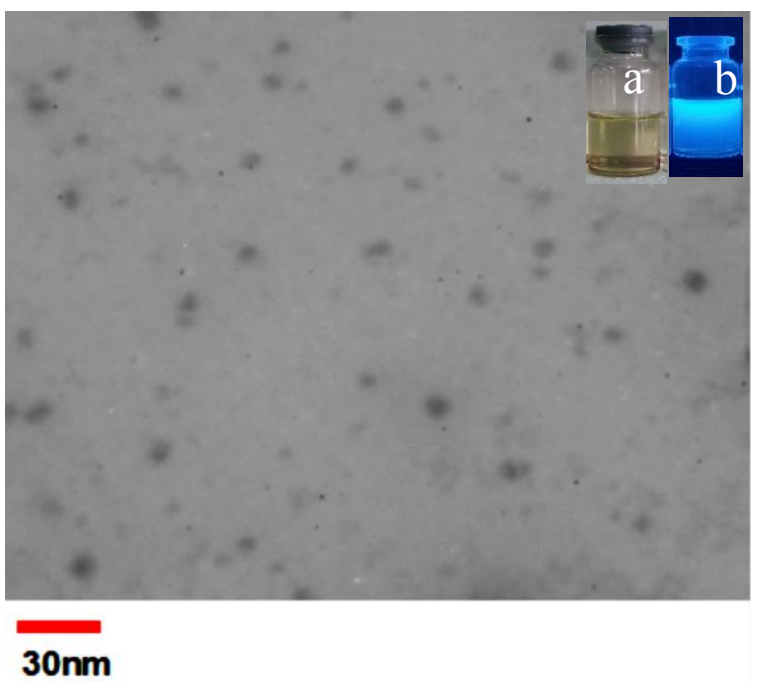

Fig 1 

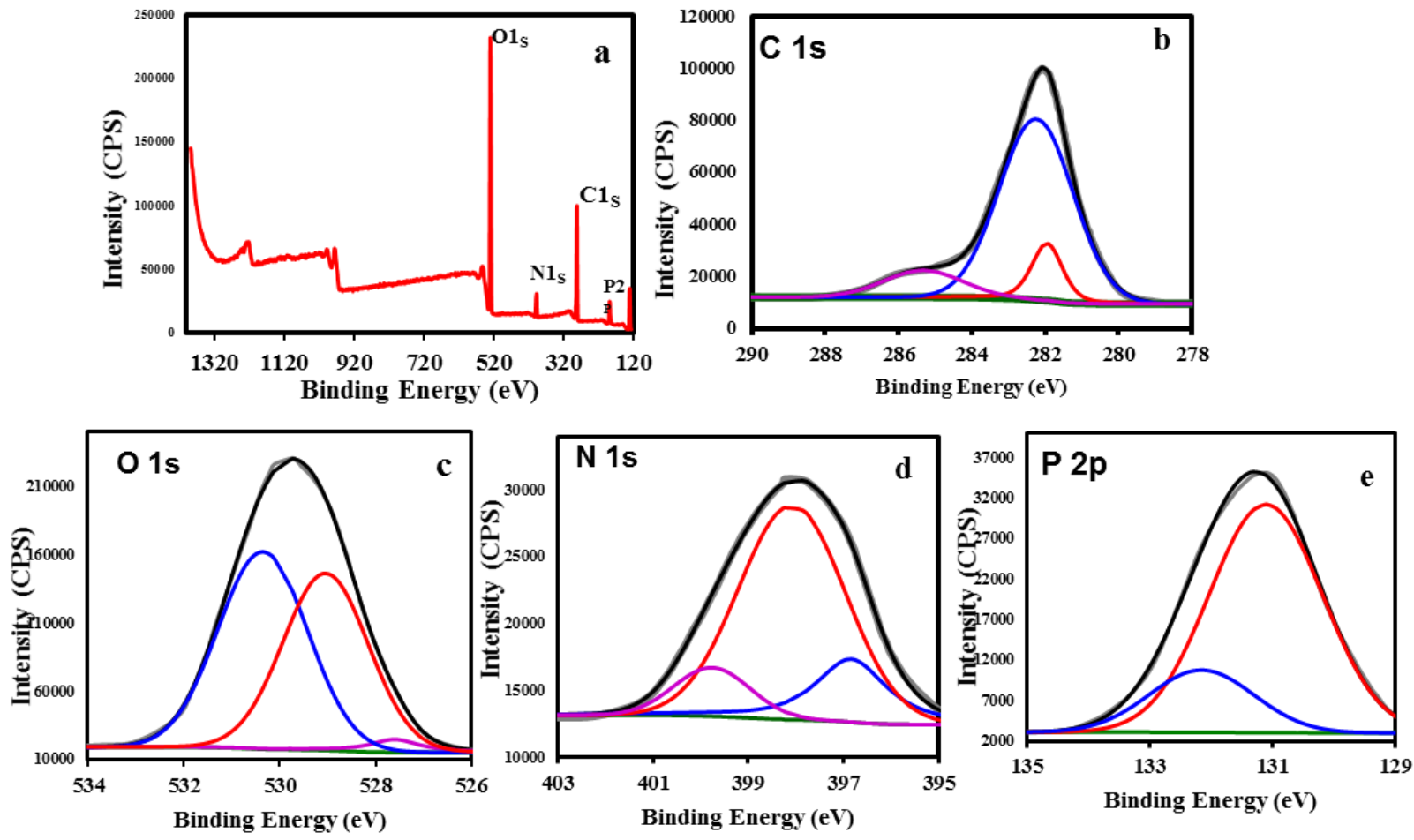

Fig 2 

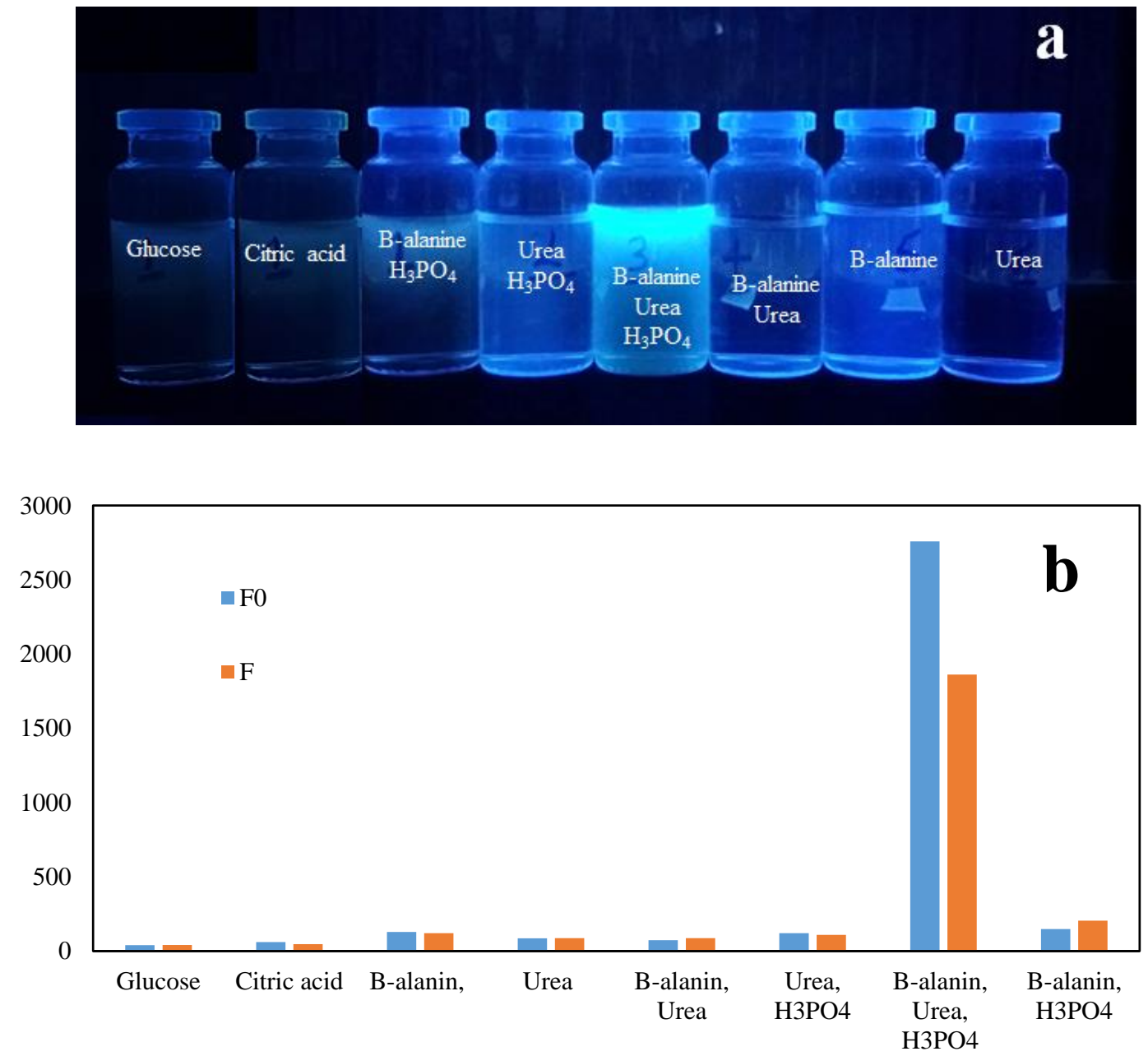

Fig 3 

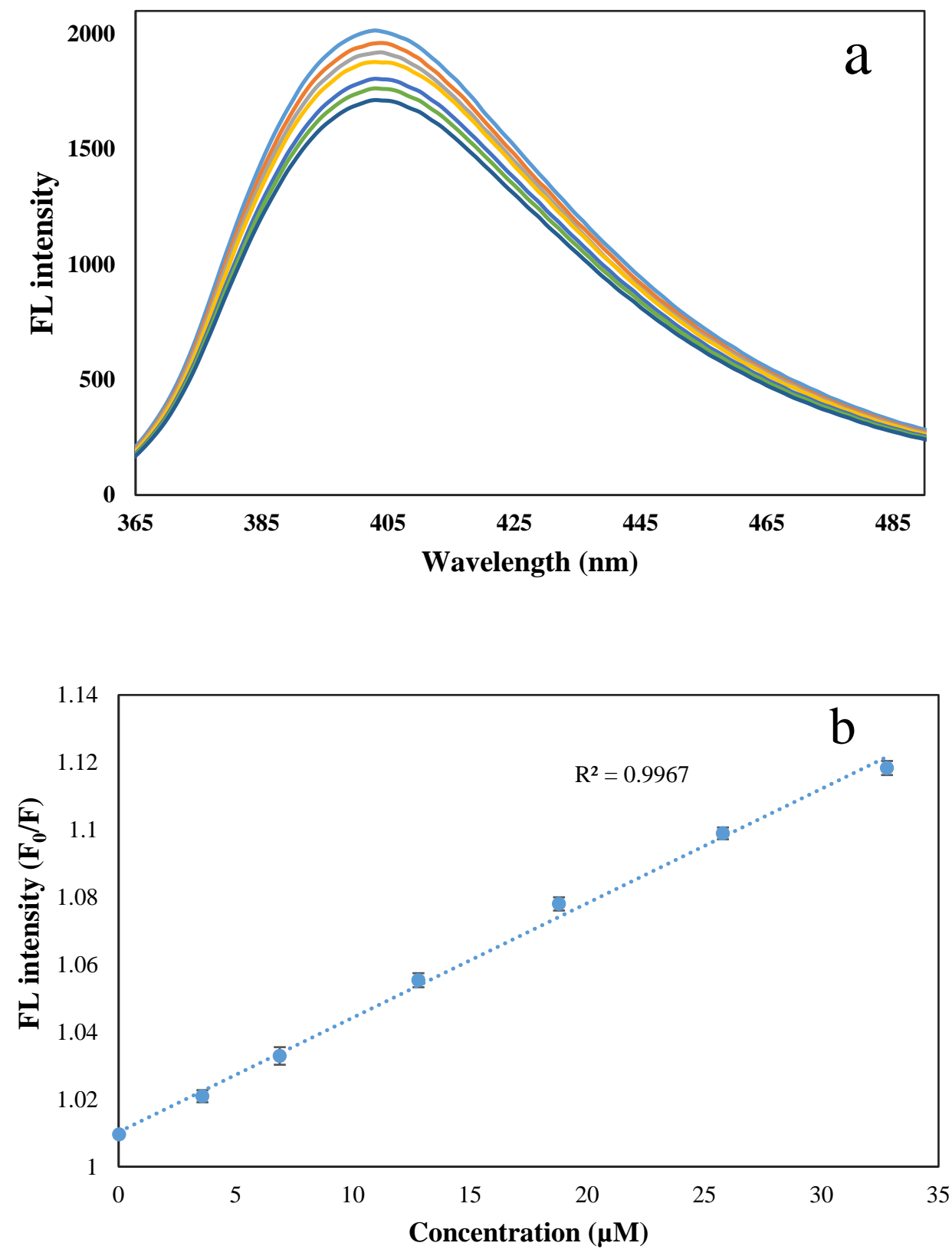

Fig 4 


\section{Graphical Index}

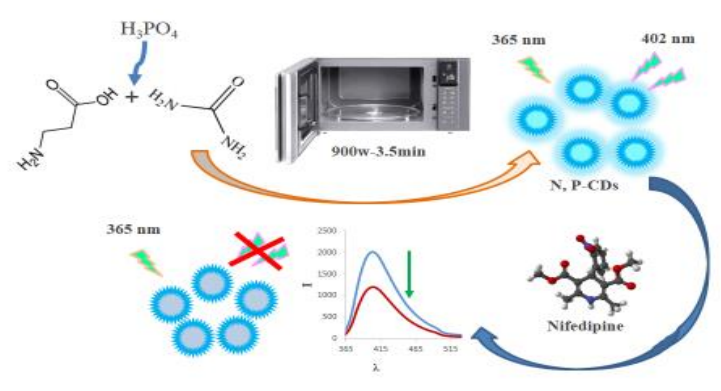

\section{References}

[1] N. Özaltin, C. Yardimci, I. Süslü, Journal of pharmaceutical and biomedical analysis, 30 (2002) 573582.

[2] M. Baghayeri, M. Namadchian, H. Karimi-Maleh, H. Beitollahi, Journal of electroanalytical chemistry, 697 (2013) 53-59.

[3] H.-J. Wang, C.-K. Lu, W.-C. Chen, A.-C. Chen, Y.-F. Ueng, journal of food and drug analysis, 27 (2019) 284-294.

[4] A.G. Ellrodt, M.J. Ault, The American journal of emergency medicine, 3 (1985) 16-24.

[5] S. Laurent, Pharmacological research, 124 (2017) 116-125.

[6] M. Gil-Agustí, S. Carda-Broch, L. Monferrer-Pons, J. Esteve-Romero, Biomedical Chromatography, 20 (2006) 154-160.

[7] M. Vertzoni, C. Reppas, H. Archontaki, Analytica chimica acta, 573 (2006) 298-304.

[8] S. Ahmed, A. Alqurshi, A.-M.I. Mohamed, Talanta, 184 (2018) 296-306.

[9] M. Khairy, A.A. Khorshed, F.A. Rashwan, G.A. Salah, H.M. Abdel-Wadood, C.E. Banks, Sensors and Actuators B: Chemical, 252 (2017) 1045-1054.

[10] J. Tian, M. Huang, Y. Yang, D. Wang, J. Lu, Biosensors and Bioelectronics, 135 (2019) 160-165.

[11] M. Hamd, A. Abdellatif, S. Derayea, O. Abdelmageed, H. Askal, Ind Chem, 1 (2015) 2.

[12] P. Tulasamma, P. Venkateswarlu, Arabian Journal of Chemistry, 9 (2016) S1603-S1609. 
[13] A. Iqbal, Y. Tian, X. Wang, D. Gong, Y. Guo, K. Iqbal, Z. Wang, W. Liu, W. Qin, Sensors and Actuators B: Chemical, 237 (2016) 408-415.

[14] R. Jalili, M. Amjadi, RSC advances, 5 (2015) 74084-74090.

[15] H. ESKALEN, S. URUŞ, Ş. ÖZĞAN, B. TAHTA, A.B. SÜNBÜL, Quantum Dots: Properties and Applications, 96 (2021) 81-94.

[16] Y. Chen, X. Shan, D. Jiang, X. Li, Analytical Sciences, (2021) $20 \mathrm{P} 433$.

[17] S. Narimani, N. Samadi, Microchemical Journal, 168 (2021) 106372.

[18] S. Lei, N. Chang, J. Zhang, H. Wang, Analytical Sciences, (2020) 20 2294.

[19] P. Zuo, X. Lu, Z. Sun, Y. Guo, H. He, Microchimica Acta, 183 (2016) 519-542.

[20] S. Miao, K. Liang, J. Zhu, B. Yang, D. Zhao, B. Kong, Nano Today, 33 (2020) 100879.

[21] C.-L. Shen, Q. Lou, K.-K. Liu, L. Dong, C.-X. Shan, Nano Today, 35 (2020) 100954.

[22] C.L. Shen, Q. Lou, C.F. Lv, J.H. Zang, S.N. Qu, L. Dong, C.X. Shan, Advanced Science, 6 (2019) 1802331.

[23] C.L. Shen, Q. Lou, J.H. Zang, K.K. Liu, S.N. Qu, L. Dong, C.X. Shan, Advanced Science, 7 (2020) 1903525.

[24] K.M. Omer, A.Q. Hassan, Microchimica Acta, 184 (2017) 2063-2071.

[25] M. Zaib, A. Akhtar, F. Maqsood, T. Shahzadi, Arabian Journal for Science and Engineering, 46 (2021) 437-446.

[26] Z. Ma, H. Ming, H. Huang, Y. Liu, Z. Kang, New Journal of Chemistry, 36 (2012) 861-864.

[27] H. Eskalen, S. Uruş, S. Cömertpay, A.H. Kurt, Ş. Özgan, Industrial Crops and Products, 147 (2020) 112209.

[28] S. Xu, S. Ye, Y. Xu, F. Liu, Y. Zhou, Q. Yang, H. Peng, H. Xiong, Z. Zhang, Analytical Sciences, (2019) $19 \mathrm{P} 350$.

[29] J.Y. Wei, Q. Lou, J.H. Zang, Z.Y. Liu, Y.L. Ye, C.L. Shen, W.B. Zhao, L. Dong, C.X. Shan, Advanced Optical Materials, 8 (2020) 1901938.

[30] D. Pan, J. Zhang, Z. Li, C. Wu, X. Yan, M. Wu, Chemical Communications, 46 (2010) 3681-3683.

[31] G.E. LeCroy, S.K. Sonkar, F. Yang, L.M. Veca, P. Wang, K.N. Tackett, J.-J. Yu, E. Vasile, H. Qian, Y. Liu, ACS nano, 8 (2014) 4522-4529.

[32] J.P. Paraknowitsch, A. Thomas, Energy \& Environmental Science, 6 (2013) 2839-2855.

[33] M.K. Barman, B. Jana, S. Bhattacharyya, A. Patra, The Journal of Physical Chemistry C, 118 (2014) 20034-20041.

[34] L. Ansari, S. Hallaj, T. Hallaj, M. Amjadi, Colloids and Surfaces B: Biointerfaces, (2021) 111743.

[35] A.B. Bourlinos, G. Trivizas, M.A. Karakassides, M. Baikousi, A. Kouloumpis, D. Gournis, A.

Bakandritsos, K. Hola, O. Kozak, R. Zboril, Carbon, 83 (2015) 173-179.

[36] S. Chandra, A.R. Chowdhuri, D. Laha, S.K. Sahu, Luminescence, 33 (2018) 336-344.

[37] D. Gu, P. Zhang, L. Zhang, H. Liu, Z. Pu, S. Shang, Optical Materials, 83 (2018) 272-278.

[38] K.M. Omer, N.N. Mohammad, S.O. Baban, Catalysis Letters, 148 (2018) 2746-2755.

[39] R. Shokri, M. Amjadi, Spectrochimica Acta Part A: Molecular and Biomolecular Spectroscopy,

246 (2021) 118951.

[40] E.F. Simões, J.M. Leitão, J.C.E. da Silva, Analytica chimica acta, 960 (2017) 117-122.

[41] S.Y. Park, H.U. Lee, E.S. Park, S.C. Lee, J.-W. Lee, S.W. Jeong, C.H. Kim, Y.-C. Lee, Y.S. Huh, J. Lee, ACS applied materials \& interfaces, 6 (2014) 3365-3370.

[42] J. Jiang, Y. He, S. Li, H. Cui, Chemical communications, 48 (2012) 9634-9636.

[43] H. Miao, L. Wang, Y. Zhuo, Z. Zhou, X. Yang, Biosensors and Bioelectronics, 86 (2016) 83-89.

[44] J.N. Hart, P.W. May, N.L. Allan, K.R. Hallam, F. Claeyssens, G.M. Fuge, M. Ruda, P.J. Heard, Journal of Solid State Chemistry, 198 (2013) 466-474.

[45] K.M. Omer, D.I. Tofiq, A.Q. Hassan, Microchimica Acta, 185 (2018) 1-8. 
